\title{
AMCoR
}

Asahikawa Medical College Repository http://amcor.asahikawa-med.ac.jp/

Parasitology (2007) 134(5):713-722.

A molecular phylogeny of the genus Echinococcus inferred from complete mitochondrial genomes

M. NAKAO ; D. P. McMANUS ; P. M. SCHANTZ ; P. S. CRAIG ; A. ITO 
Submitted to Parasitology by Nakao M with 4 figures and 3 tables

\section{A molecular phylogeny of the genus Echinococcus inferred from complete mitochondrial genomes}

M. NAKAO ${ }^{1 *}$, D. P. McMANUS ${ }^{2}$, P. M. SCHANTZ ${ }^{3}$, P. S. CRAIG ${ }^{4}$ and A. ITO ${ }^{1}$

${ }^{1}$ Department of Parasitology, Asahikawa Medical College, Asahikawa, Hokkaido 078-8510, Japan

${ }^{2}$ Molecular Parasitology Laboratory, The Queensland Institute of Medical Research and the University of Queensland, Brisbane, Queensland 4029, Australia

${ }^{3}$ Division of Parasitic Diseases, National Centers for Infectious Diseases, Centers for Disease Control and Prevention, Atlanta, GA 30341, USA

${ }^{4}$ Cestode Zoonoses Research Group, Bioscience Research Institute and School of Environment and Life Sciences, University of Salford, Great Manchester M5 $4 W T, U K$

Running title: Phylogeny of Echinococcus

* Corresponding author: Department of Parasitology, Asahikawa Medical College, Asahikawa, Hokkaido 078-8510, Japan. Tel: +81 16668 2422. Fax: +81 16668 2429. E-mail: nakao@asahikawa-med.ac.jp 


\section{SUMMARY}

Taxonomic revision by molecular phylogeny is needed to categorize members of the genus Echinococcus (Cestoda: Taeniidae). We have reconstructed the phylogenetic relationships of E. oligarthrus, E. vogeli, E. multilocularis, E. shiquicus, E. equinus, E. ortleppi, E. granulosus sensu stricto and 3 genotypes of $E$. granulosus sensu lato (G6, G7 and G8) from their complete mitochondrial genomes. Maximum likelihood and partitioned Bayesian analyses using concatenated data sets of nucleotide and amino acid sequences depicted phylogenetic trees with the same topology. The 3 E. granulosus genotypes corresponding to the camel, pig, and cervid strains were monophyletic, and their high level of genetic similarity supported taxonomic species unification of these genotypes into E. canadensis. Sister species relationships were confirmed between $E$. ortleppi and E. canadensis, and between $E$. multilocularis and $E$. shiquicus, regardless of the analytical approach employed. The basal positions of the phylogenetic tree were occupied by the neotropical endemic species, $E$. oligarthrus and E. vogeli, whose definitive hosts are derived from carnivores that immigrated from North America after the formation of the Panamanian land bridge. Host-parasite co-evolution comparisons suggest that the ancestral homeland of Echinococcus was North America or Asia, depending on whether the ancestral definitive hosts were canids or felids.

Key words: Echinococcus, mitochondrial genome, phylogeny, taxonomic revision. 


\section{INTRODUCTION}

Cestodes of the family Taeniidae are parasitic in mammals and are placed within either of 2 genera, Taenia and Echinococcus (Hoberg et al. 2000; Kumaratilake and Thompson, 1982). The adult tapeworms live in the digestive tract of their definitive hosts, while the bladderlike larvae occur in the internal organs of intermediate hosts. The predator-prey relationships of the mammalian hosts perpetuate the life cycle of the taeniid cestodes. The tiny adult tapeworms of the genus Echinococcus utilize canids and/or felids as definitive hosts, and their larvae develop into hydatid cysts in intermediate hosts including ungulates, rodents and humans. The 2 main species, E. granulosus and E. multilocularis, cause cystic and alveolar echinococcosis in humans, respectively. The organisms of Echinococcus have a biphasic propagation manner comprising a sexual reproductive phase in the hermaphroditic adults and an asexual proliferation (of protoscoleces) at the larval stage. The self fertilization of the adults and the clonal amplification of the larvae are responsible for genetic monomorphism within isolated Echinococcus populations (Lymbery and Thompson, 1996; Haag et al. 1999), and may lead, eventually, to the speciation of Echinococcus (Smyth and Smyth, 1964).

The classification of Echinococcus has been controversial because of the paucity of phenotypic characters and inadequate taxonomic descriptions. In taxonomic terms, many species and subspecies of Echinococcus were originally described, based mainly on host-parasite specificity characteristics (Ortlepp, 1937; Lopez-Neyra and Soler Planas, 1943; Williams and Sweatman, 
1963; Verster, 1965). However, most of these taxa were regarded as synonyms for E. granulosus (Rausch, 1967), and the subsequent reclassification permitted only 4 morphospecies, namely E. granulosus, E. multilocularis, E. oligarthrus and E. vogeli (Rausch and Bernstein, 1972). The biological variants of E. granulosus, which utilize particular ungulates as intermediate hosts, have been designated as strains (Thompson et al. 1995). Molecular genetic studies, using mainly mitochondrial DNA (mtDNA) sequences, supported the strain definition and identified 10 genotypes (G1 to G10) within E. granulosus (Bowles and McManus, 1994; Bowles et al. 1995; Scott et al. 1997; Lavikainen et al. 2003). The taxonomic revision of Echinococcus is now required to determine the status of sibling or cryptic species. To date, reviving the names of synonyms, the oversimplified E. granulosus has been split into E. granulosus sensu stricto (genotypes G1 to G3), E. equinus (G4) and E. ortleppi (G5), but the species status of genotypes G6 to $\mathrm{G} 10$ is still ambiguous (Thompson and McManus, 2003). Most recently, a new sibling species, E. shiquicus, whose adult is morphologically similar to E. multilocularis, has been found in Tibet (Xiao et al. 2005).

Mitochondrial DNA (mtDNA) has more power than nuclear DNA in reconstructing phylogenetic relationships among closely related species because of the rapid sequence evolution (Brown et al. 1979), and large data sets derived from mitochondrial genomes have the potential for resolving problematic issues in taxonomy (Mueller et al. 2004). In this study, the phylogeny of the genus Echinococcus was clarified by using the complete mitochondrial genomes of representative taxa in order to improve the consistency between species 
status and traditional nomenclatures. The concatenated data sets of nucleotide and amino acid sequences derived from all mitochondrial genes were analyzed with an array of phylogenetic methods including maximum likelihood and partitioned Bayesian analyses. In particular, the latter is the only method that can be used to analyze a large data set by incorporating complex substitution models into the statistical process (Ronquist et al. 2003; Nylander et al. 2004). Also, the ancestral origin of Echinococcus was inferred, based on the phylogeny of Echinococcus and the evolutionary history of mammalian host animals.

\section{MATERIALS AND METHODS}

Taxon sampling

Ten taxa of Echinococcus and 1 outgroup (Taenia solium) were used in this study (Table 1). Complete published sequences were available for mitochondrial genomes of $E$. granulosus sensu stricto, E. equinus, $E$. multilocularis and T. solium (Le et al. 2002; Nakao et al. 2002b, 2003). In addition, complete mtDNA sequences were obtained for larval isolates of $E$. oligarthrus (Panama), E. vogeli (Colombia), E. shiquicus (Tibet), E. ortleppi (Argentina), E. granulosus genotype G6 (Kazakhstan), E. granulosus genotype G7 (Poland) and E. granulosus genotype G8 (USA). Previous studies (Bowles et al. 1995; Lavikainen et al. 2003) indicate that the taxa of Echinococcus examined in this study cover a range of major sequence variation in mtDNA within the genus. 


\section{DNA sequencing}

Genomic DNA purified from each of the Echinococcus isolates was used as a template for the polymerase chain reaction (PCR). As reported previously (Nakao et al. 2003), DNA fragments $(0.7-2.5 \mathrm{~kb})$ covering complete mitochondrial genomes were amplified by PCR, and their DNA sequences were compiled into a total sequence. In brief, the initial amplification of mtDNA was carried out using primers designed from the conserved regions of $E$. multilocularis mtDNA (Nakao et al. 2002b). The sequences of these amplicons allowed us to design additional primers, whereby the remaining unknown regions were amplified. The cycle sequencing kits, DYEnamic ET terminator (Amersham Biosciences) and BigDye terminator (Applied Biosystems), were used for the direct sequencing of PCR products. After each sequencing reaction, synthesized ladders were run on an ABI PRISM 377 genetic analyzer (Applied Biosystems). Large DNA templates were sequenced by primer walking.

\section{Data processing}

Mitochondrial genomes were annotated as reported previously (Nakao et al. 2002b). Open reading frames (ORFs) of 12 protein-coding genes for ATPase subunit 6 (atp6), cytochrome $b$ (cob), cytochrome $c$ oxidase subunits 1 to 3 (cox1 to 3 ) and NADH dehydrogenase subunits 1 to 6 and $4 \mathrm{~L}$ (nad1 to 6 and nad4L) were determined using the echinoderm mitochondrial genetic code (Nakao et al. 2000; Telford et al. 2000). Large and small subunit rRNA genes ( $r r n L$ and $r r n S)$ and 22 tRNA genes (trn) were identified by their sequence similarities and 
secondary structures. Multiple alignments and basic statistics of nucleotide and amino acid sequences were achieved by ClustalX 1.82 (Thompson et al. 1997) and MacClade 4.08 (Maddison and Maddison, 2000). PAUP4.0b10 (Swofford, 2002) was applied to make pairwise distance matrices. The conservative regions of mitochondrial genomes were displayed by the program plotcon of EMBOSS 3.00 (Rice et al. 2000) using the alignment data of complete nucleotide sequences from all Echinococcus taxa. Gene-by-gene alignment was carried out for the 11 taxa (10 Echinococcus and 1 Taenia) to prepare data sets for phylogenetic analyses. Based on the alignments of deduced amino acid sequences, the nucleotide sequences of each protein-coding gene were aligned manually. The alignments of rRNA and tRNA genes were corrected by deleting ambiguous positions, such as loops and indels. The resultant alignments were concatenated into 3 data sets as follows: data set \#1 comprising nucleotide sequences for all genes (total 13,130 sites); data set \#2 comprising nucleotide sequences for 12 protein-coding genes excluding the third codon position (total 6,746 sites); and data set \#3 of amino acid sequences for the 12 proteins (total 3,361 sites). Non-coding regions were considered unusable for phylogenetic analyses because they contained highly repetitive sequences.

PAUP4.0b10 (Swofford, 2002) was employed for maximum likelihood $(\mathrm{ML})$ analysis using the nucleotide data sets \#1 and \#2. A suitable nucleotide substitution model and its parameter values were determined by Akaike Information Criterion (AIC) implemented in MODELTEST 3.7 (Posada and Crandall, 1998). A full heuristic search algorithm was utilized to estimate the 
ML trees. To conduct ML analysis for proteins, the program proml of PHYLIP 3.6 (Felsenstein, 2005) was run using the amino acid data set \#3. The Jones-Taylor-Thornton (JTT) model for the substitution of amino acids was used with a gamma shape parameter estimated from the data. In both analyses, the robustness of inferred trees was tested by non-parametric bootstrapping with 1,000 pseudoreplicates. Consensus trees were constructed by the $50 \%$ majority rule and rooted with an outgroup taxon.

Partitioned Bayesian analyses were implemented using MrBayes 3.1 (Ronquist and Huelsenbeck, 2003). The nucleotide data set \#1 comprising all genes was divided into 15 partitions. Twelve protein-coding genes and two rRNA genes were treated as individual data partitions and all tRNA genes were assigned as a single data partition. The best-fit nucleotide substitution models of each partition were selected by AIC implemented in MrModeltest 2.2 (modified from MODELTEST by J. A. A. Nylander, Uppsala University, Sweden). The amino acid data set \#3 comprising all inferred proteins was divided into 12 partitions and their substitution models were selected by AIC in PROTTEST 1.26 (Abascal et al. 2005). Using each of the partitioned data sets, a Metropolis-coupled Markov chain Monte Carlo analysis was run for 1 million generations to estimate the posterior probabilities of trees. The tree sampling was taken every 1,000 generations and all tree samples before the chain reached a stationarity were discarded as burn-in. Two independent analyses were conducted to verify the consistency of results.

RESULTS 


\section{Characterization of mitochondrial genomes}

All mtDNAs examined in this study were in the form of a covalently closed-circular molecule of $\sim 14$ kilo base pairs (Table 1). The genomes comprised 12 protein-coding genes, 2 rRNA genes, 22 tRNA genes ( 1 for each of 18 amino acids and 2 each for serine and leucine) and 2 short non-coding regions involved in replication and translation. The gene for ATPase subunit 8 was absent. Most of the genes were separated by a few bases, and the protein-coding genes contained no introns. The gene orders were identical among all of the genomes examined. All genes were located on the same strand, which was strongly biased towards thymine and against cytosine (Table 1). The nucleotide A+T contents of the Echinococcus mtDNAs ranged between 67.1 and $69.3 \%$. The protein-coding genes were initiated by either an ATG or GTG codon and terminated by a TAG or TAA codon. The abbreviated stop codons $\mathrm{T}$ and $\mathrm{TA}$, which are modified into a complete TAA codon by polyadenylation, were detected in the Taenia mtDNA (Nakao et al. 2003) but not in the Echinococcus mtDNAs.

The similarity scores of complete nucleotide sequences among 10 Echinococcus mtDNAs were plotted for every nucleotide position (Fig. 1). Conservative sequences were demonstrated in cob, nad3, cox1, cox2 and rrnS, while the non-coding region, located upstream from nad5, was highly polymorphic due to the deletion and insertion of repetitive sequences. An extreme imbalance of the similarity scores was observed within the sequences for nad5 and rrnL. Concerning the tRNA genes, the sequences of the 
anticodon arm (17 bases for each tRNA) were well conserved. However, the loops of the $\mathrm{D}$ and $\mathrm{T} \Psi \mathrm{C}$ arms were variable (data not shown).

For all Echinococcus taxa, the number of nucleotide substitutions in all protein-coding genes was counted at every codon position after the alignments of each gene were concatenated. Of a total of 3,426 variable sites, the second codon position had the fewest sites ( $n=426,12.4 \%$ ), followed by the first codon position ( $n=904,26.4 \%$ ), whereas the third codon position had the most sites ( $n$ $=2,096,61.2 \%)$. The transition/transversion ratios for the first, second and third codon positions were 2.55, 1.68 and 1.69, respectively. The average frequencies of amino acid replacements are illustrated by MacClade's bubble charts using the amino acid data set \#3 of all proteins (Fig. 2). The most frequent amino acid changes among the mitochondrial proteins predicted for the Echinococcus taxa were Val to Ile, Ile to Val, Leu to Phe and Phe to Leu. Most of these changes corresponded directly to the transitional substitutions of the first codon position.

\section{Phylogenetic analyses}

The phylogenetic relationships among 10 Echinococcus taxa were estimated mainly by using the nucleotide data set \#1 representing all of the mitochondrial genes. To reduce the influence of saturation at the third codon position, the nucleotide data set \#2 of 12 protein-coding genes (without third codon positions) and the amino acid data set \#3 of 12 proteins were also used. The substitution models of these data sets for ML and partitioned Bayesian analyses are shown in Table 2. The Tamura-Nei $(\operatorname{TrN})+\mathrm{G}+\mathrm{I}$ model was selected for $\mathrm{ML}$ analysis 
as the best fit model to describe the data set \#1. Parameters for this model included base frequencies $(A=0.2114, C=0.0653, G=0.2340$ and $T=0.4893)$, substitution rate matrix $(A C=1.0000, A G=11.6176, A T=1.0000, C G=1.0000$, $\mathrm{CT}=8.3353$ and $\mathrm{GT}=1.0000)$, gamma distribution shape (0.9340) and proportion of invariable sites $(0.4179)$. The tree topology inferred from the $M L$ analysis is displayed as a cladogram (Fig. 3A) and as a phylogram (Fig. 3B). Two clades ( $\alpha$ and $\beta$ ) were supported with high bootstrap values (98 - 100\%). Clade $\alpha$ included $E$. ortleppi and genotypes G6, G7 and G8, whereas clade $\beta$ consisted of $E$. multilocularis and $E$. shiquicus. The phylogram depicted a close relationship between the members of clade $\alpha$. The other taxa, E. oligarthrus, $E$. vogeli, E. granulosus sensu stricto and E. equinus, were divided deeply into independent branches with moderate bootstrap values $(71-89 \%)$. Further ML analyses using the data sets \#2 and \#3 produced phylogenies with unresolved nodes (Fig. 3A). In trees derived from both data sets, the basal positions of $E$. oligarthrus and E. vogeli were strongly supported, whereas the phylogenetic relationships among $E$. granulosus sensu stricto, $E$. equinus and clades $\alpha$ and $\beta$ were uncertain because their nodes showed low bootstrap values. Partitioned Bayesian analyses using data sets \#1 and \#3 resulted in a robust phylogeny (Fig. 3A). The posterior probabilities of each node mostly reached $100 \%$ for both data sets. The tree topology of the Bayesian analyses was consistent with those of the ML analyses. The Bayesian analyses supported a sister group relationship between clades $\alpha$ and $\beta$. Furthermore, the resultant trees clarified the phylogenetic positions of E. granulosus sensu stricto and E. equinus, which remained as uncertain in the $\mathrm{ML}$ analyses. 
Host-parasite relationships

Mammalian hosts for the menbers of Echinococcus were mapped to the parasite phylogram to examine host-parasite relationships (Fig. 3B). The definitive hosts were represented by the families Felidae and Canidae. The orders Rodentia, Artiodactyla, Perissodactyla and Lagomorpha were used to simplify an abundance of the intermediate hosts. A switch in definitive hosts might occur in the basal node of the phylogram because only E. oligarthrus utilizes Felidae, but the other monophyletic taxa colonize Canidae including dogs and foxes. Clade $\beta$ consists of $E$. multilocularis and E. shiquicus which especially colonize foxes. Ancestral definitive hosts, whether Felidae or Canidae, were uncertain. Concerning intermediate hosts, a coevolutionary relationship was evident in clade $\alpha$ consisting of E. ortleppi and genotypes G6, G7 and G8. The members of the clade colonize Artiodactyla including cattle (E. ortleppi), camels (G6), pigs (G7) and cervids (G8). The basal species of E. oligarthrus and E. vogeli are associated typically with Rodentia, suggesting that rodents might be their ancestral intermediate hosts.

\section{Divergence of closely related taxa}

Pairwise divergence values for mtDNA sequences were compared to evaluate the species status of E. ortleppi and genotypes G6, G7 and G8. Within the family Taeniidae, Taenia saginata and Taenia asiatica are recognized as a pair of recently evolved species (Hoberg et al. 2001) and are useful as a reference for closely related species. The complete nucleotide sequences of the cob and 
cox1 genes have been determined for T. saginata and T. asiatica (Nakao et al. 2002a). Therefore, these genes were selected for comparisons of pairwise divergence. The matrix of Kimura's 2-parameter distance was calculated from the concatenated data set for the $c o b$ and $\operatorname{cox} 1$ sequences (total 2,676 sites) using a gamma shape parameter $(a=1)$. The pairwise divergence value between $T$. saginata and $T$. asiatica was used as a standard for incipient speciation. As shown in Table 3, the remarkably low value of pairwise divergence between G6 and G7 suggests that these genotypes belong to a single species. On the other hand, the species status of G8 is problematic, because the divergence values between G8 and the other genotypes were close to the threshold value between $T$. saginata and $T$. asiatica. The divergence values between $E$. ortleppi and the 3 genotypes were at interspecific levels.

\section{DISCUSSION}

Concurrent descriptions of species, strains and genotypes have been used to record the biodiversity of Echinococcus in the context of medical and veterinary criteria for diagnosing and controlling echinococcosis in various settings (McManus and Thompson, 2003; Thompson and McManus, 2003). As a general rule, strains and genotypes are employed to categorize intraspecific variation. However, previous molecular phylogenetic studies on Echinococcus (Bowles et al. 1995; Thompson et al. 1995) demonstrated that all strains or genotypes of $E$. granulosus could not be assembled into a monophyletic group, indicating that E. granulosus sensu lato is a cryptic species complex. 
Accordingly, the arbitrary use of strains and genotypes without a correction of the old nomenclature causes unnecessary confusion. The taxonomy of the cryptic species should be based entirely on their phylogenetic relationships, and appropriate scientific names should be used instead of strains and genotypes. The present study provides useful information for dividing E. granulosus sensu lato into distinct species.

The phylogeny of Echinococcus presented in this study is the first to be reconstructed from whole mitochondrial genome sequences. Confidence in the proposed topology was increased by the overall support of the nodes with ML and partitioned Bayesian analyses. However, divergence dates cannot be estimated due to the absence of fossil records. The resultant phylogeny included representative genotypes of E. granulosus sensu lato. Genotypes G2 (Tasmanian sheep strain) and G3 (buffalo strain) were not used in this study because they have very similar mtDNA sequences to that of genotype $\mathrm{G} 1$ (sheep strain) (Bowles et al. 1995). Thompson et al. (1995) regarded the genotypes G1, G2 and G3 as a single species, and these constitute the species E. granulosus sensu stricto. The present phylogeny confirms the validity of $E$. granulosus sensu stricto and supports the recent revisions of genotypes G4 (horse strain) and G5 (cattle strain) into E. equinus and E. ortleppi, respectively (Thompson and McManus, 2003). Moreover, the tree depicted a monophyly of E. ortleppi and genotypes G6 (camel strain), G7 (pig strain) and G8 (cervid strain). The other genotypes G9 (Polish human strain) and G10 (Fennoscandian cervid strain) were not analyzed in this study. However, close relationships between $\mathrm{G} 7$ and $\mathrm{G} 9$ and between $\mathrm{G} 8$ and $\mathrm{G} 10$ have been reported 
using mtDNA sequences (Scott et al. 1997; Lavikainen et al. 2003; Xiao et al. 2005; Thompson et al. 2006). In this study, the values of pairwise divergence among G6, G7 and G8 were lower than a threshold value for speciation estimated from $T$. saginata and T. asiatica. Using multiple loci of mtDNA (nad1, atp6 and cox1), Lavikainen et al. (2006) showed that the camel, pig and cervid strains clustered into a monophyletic group and that the cervid genotypes (G8 and G10) could not be separated out from this group. This result and our data suggest that the camel, pig and cervid strains may belong to a single species. The same suggestion has been made by Xiao et al. (2005) and Thompson et al. (2006). The camel and pig strains have not previously been named (Thompson et al. 1995), but the cervid strain was formerly named E. granulosus canadensis (Cameron, 1960; Webster and Cameron, 1961) or E. granulosus borealis (Sweatman and Williams, 1963). In terms of priority for nomenclature, $E$. canadensis is the most suitable for genotypes G6 to G10. Based on the resultant phylogeny, we recommend a taxonomic revision to unify these genotypes into E. canadensis. Lavikainen et al. (2005) have already made the same proposal. In our recommendation, E. canadensis and E. ortleppi are regarded as 'sister species'. An alternative revision to this unified classification is to describe a new species for the camel and pig strains (genotypes G6, G7 and G9). In this case, the use of E. canadensis would be limited to the cervid strain (genotypes G8 and G10). The morphological similarities between the camel and pig strains (Eckert et al. 1993) provide a significant piece of evidence to support the new species. Further, comprehensive population genetic, morphological and ecological studies are required for a final decision about the 
taxonomic status of the camel, pig and cervid strains.

A sister species relationship between E. multilocularis and E. shiquicus was demonstrated in this study. The morphological similarities between both adult tapeworms (Xiao et al. 2005) and the common usage of foxes as definitive hosts support this relationship. The extreme exogenous proliferation of larval cysts is characteristic of E. multilocularis and is responsible for the serious pathological effects associated with alveolar echinococcosis. In contrast, the larval cyst of E. shiquicus is basically unilocular (Xiao et al. 2005). The relationship between $E$. multilocularis and $E$. shiquicus strongly suggests that $E$. multilocularis only acquired the ability of the exogenous proliferation during the bifurcation of species. Although the larvae of E. oligarthrus and E. vogeli are also polycystic, the present phylogeny indicates that both species are not related to E. multilocularis in the evolutionary process of acquiring exogenous proliferation.

The most striking feature of the present phylogeny is that basal positions are occupied by E. oligarthrus and E. vogeli. A previous study using partial nucleotide sequences of mitochondrial genes (cox1 and nad1) also ranked these species as basal lineages, but the result was not fully supported due to the short sequences examined (Bowles et al. 1995). The basal positions of $E$. oligarthrus and E. vogeli are important in considering phylogeographic patterns because both species are endemic to the Neotropical region and colonize wildlife as definitive and intermediate hosts. As compared with these endemic species, E. granulosus sensu stricto, E. equinus, E. ortleppi and E. canadensis are inadequate for phylogeographical considerations because worldwide 
livestock movement in historic and prehistoric times seriously disturbed their original distributions. Nevertheless, all mammals, which can serve as definitive and intermediate hosts for Echinococcus, are restricted to members of the superorders Laurasiatheria and Euarchontoglires of the Northern Hemisphere origin (Murphy et al. 2001), suggesting that the adaptive radiation of the mammals is linked with the evolution of Echinococcus.

We propose plausible hypotheses for the history of Echinococcus based on geological events and the evolution of felids and canids. Several neotropical felids such as jaguarondi, ocelot and jaguar are definitive hosts for E. oligarthrus, whereas only the bush dog is known to be a definitive host for E. vogeli (D'Alessandro et al. 1981; Rausch and Bernstein, 1972). As shown in Fig. 4, these carnivores are derived from immigrants from North America after the formation of the Panamanian land bridge. The immigration of North American felids and canids occurred during the early Pleistocene and these evolved into endemic species in South America (Marshall et al. 1982). Accordingly, it seems likely that the ancestral species of Echinococcus invaded South America together with the immigrants. The present members of Echinococcus usually utilize canids as definitive hosts except for E. oligarthrus. In this study, it was uncertain whether the ancestral definitive hosts were felids or canids. If they were canids, one could speculate that North America was the cradle of Echinococcus, as canids originated in North America (Wayne et al. 1997; Wang et al. 2004). Another plausible scenario should be considered in case that the ancestral hosts were felids. Recently, a large-scale molecular phylogenetic study on felids suggested that modern felids arose in Asia (Johnson et al. 2006), 
and their lineages migrated repeatedly between Eurasia and North America and invaded Africa (Fig. 4). Considering felid evolution, Asia should also be considered as a candidate for the cradle of Echinococcus.

In Africa, E. granulosus sensu stricto, E. ortleppi and E. canadensis (genotype G6) prevail in domesticated animals (McManus and Thompson, 2003), whereas Echinococcus infections of wildlife including lions, zebras, wildebeest and warthogs suggest a distinst species or strain. This parasite was formerly named as E. felidis (Ortlepp, 1937; Verster, 1965) and now categorized as the lion strain of E. granulosus sensu lato (Thompson and McManus, 2003). The genetic makeup of this strain is completely unknown because wildlife preservation currently precludes its sampling and molecular analysis. Further studies are required to clarify the taxonomic status of the lion strain of $E$. granulosus sensu lato. The molecular phylogeny of Echinococcus should be tested further by adding this taxon.

This work was supported by a Grant-in-Aid for Scientific Research from the Japan Society for Promotion of Science (14256001 and 17256002) to Akira Ito and the National Institutes of Health, Ecology of Infectious Diseases Program (1R01 TW01565-01; Principal Investigator, Philip S. Craig). We thank many colleagues and field workers who collected valuable specimens, especially Jiamin Qiu for collecting Tibetan E. shiquicus. 


\section{REFERENCES}

Abascal, F., Zardoya, R. and Posada, D. (2005). ProtTest: selection of best-fit models of protein evolution. Bioinformatics 21, 2104-2105.

Bowles, J., Blair, D. and McManus, D. P. (1994). Molecular genetic characterization of the cervid strain ('northern form') of Echinococcus granulosus. Parasitology 109, 215-221.

Bowles, J., Blair, D. and McManus, D. P. (1995). A molecular phylogeny of the genus Echinococcus. Parasitology 110, 317-328.

Brown, W. M., George, M. Jr. and Wilson, A. C. (1979). Rapid evolution of animal mitochondrial DNA. Proceedings of the National Academy of Sciences USA 76, 1967-1971.

Cameron, T. W. M. (1960). The incidence and diagnosis of hydatid cyst in Canada. Echinococcus granulosus var. canadensis. Parassitologia 2, 381-390.

D'Alessandro, A., Rausch, R. L., Morales, G. A., Collet, S. and Angel, D. (1981). Echinococcus infections in Colombian animals. The American Journal of Tropical Medicine and Hygiene 30, 1263-1276.

Eckert, J., Thompson, R. C. A., Lymbery, A. J., Pawlowski, Z. S., Gottstein, B. and Morgan, U. M. (1993). Further evidence for the occurrence of a distinct strain of Echinococcus granulosus in European pigs. Parasitology Research 79, 42-48.

Felsenstein, J. (2005). PHYLIP (Phylogeny Inference Package) version 3.6. Distributed by the author via the internet. Department of Genome Sciences, 
University of Washington, Seattle, Washington, USA.

Haag, K. L., Araujo, A. M., Gottstein, B., Siles-Lucas, M., Thompson, R. C. A. and Zaha, A. (1999). Breeding systems in Echinococcus granulosus (Cestoda; Taeniidae): selfing or outcrossing? Parasitology 118, 63-71.

Hoberg, E. P., Jones, A., Rausch, R. L., Eom, K. S. and Gardner, S. L. (2000). A phylogenetic hypothesis for species of the genus Taenia (Eucestoda: Taeniidae). The Journal of Parasitology 86, 89-98.

Hoberg, E. P., Alkire, N. L., de Queiroz, A. and Jones, A. (2001). Out of Africa: origins of the Taenia tapeworms in humans. Proceedings of the Royal Society B: Biological Sciences 268, 781-787.

Johnson, W. E., Eizirik, E., Pecon-Slattery, J., Murphy, W. J., Antunes, A., Teeling, E. and O'Brien, S. J. (2006). The late Miocene radiation of modern Felidae: a genetic assessment. Science 311, 73-77.

Kumaratilake, L. M. and Thompson, R. C. A. (1982). A review of the taxonomy and speciation of the genus Echinococcus Rudolphi 1801. Zeitschrift für Parasitenkunde 68, 121-146.

Lavikainen, A., Lehtinen, M. J., Ågren, E. and Meri, S. (2005). Phylogeny of the Fennoscandian cervid strain of Echinococcus granulosus. Bulletin of the Scandinavian-Baltic Society for Parasitology 14, 92.

Lavikainen, A., Lehtinen, M. J., Meri, T., Hirvela-Koski, V. and Meri, S. (2003). Molecular genetic characterization of the Fennoscandian cervid strain, a new genotypic group (G10) of Echinococcus granulosus. Parasitology 127, 207-215.

Lavikainen, A., Lehtinen, M. J., Laaksonen, S., Ågren, E., Oksanen, A. and 
Meri, S. (2006). Molecular characterization of Echinococcus isolates of cervid origin from Finland and Sweden. Parasitology - IN PRESS.

Le, T. H., Pearson, M. S., Blair, D., Dai, N., Zhang, L. H. and McManus, D. P. (2002). Complete mitochondrial genomes confirm the distinctiveness of the horse-dog and sheep-dog strains of Echinococcus granulosus. Parasitology 124, 97-112.

Lopez-Neyra, C. R. and Soler Planas, M. A. (1943). Revision del genero Echinococcus Rud y description de una especie nuéva Parasita intestinal del perro en Almeria. Revista ibérica de parasitología 3, 169-194.

Lymbery, A. J. and Thompson, R. C. A. (1996). Species of Echinococcus: pattern and process. Parasitology Today 12, 486-491.

Maddison, D. R. and Maddison, W. P. (2000). MacClade 4: analysis of phylogeny and character evolution. Sinauer Associates, Sunderland, Massachusetts, USA.

Marshall, L. G., Webb, S. D., Sepkoski, J. J. and Raup, D. M. (1982). Mammalian evolution and the Great American Interchange. Science 215, 1351-1357.

McManus, D. P. and Thompson, R. C. A. (2003). Molecular epidemiology of cystic echinococcosis. Parasitology 127 (Suppl.), S37-S51.

Mueller, R. L., Macey, J. R., Jaekel, M., Wake, D. B. and Boore, J. L. (2004). Morphological homoplasy, life history evolution, and historical biogeography of plethodontid salamanders inferred from complete mitochondrial genomes. Proceedings of the National Academy of Sciences USA 101, 13820-13825.

Murphy, W. J., Eizirik, E., O'Brien, S. J., Madsen, O., Scally, M., Douady, C. 
J., Teeling, E., Ryder, O. A., Stanhope, M. J., de Jong, W. W. and Springer,

M. S. (2001). Resolution of the early placental mammal radiation using Bayesian phylogenetics. Science 294, 2348-2351.

Nakao, M., Okamoto, M., Sako, Y., Yamasaki, H., Nakaya, K. and Ito, A. (2002a). A phylogenetic hypothesis for the distribution of two genotypes of the pig tapeworm Taenia solium worldwide. Parasitology 124, 657-662.

Nakao, M., Sako, Y. and Ito, A. (2003). The mitochondrial genome of the tapeworm Taenia solium: a finding of the abbreviated stop codon $\mathrm{U}$. The Journal of Parasitology 89, 633-635.

Nakao, M., Sako, Y., Yokoyama, N., Fukunaga, M. and Ito, A. (2000). Mitochondrial genetic code in cestodes. Molecular and Biochemical Parasitology 111, 415-424.

Nakao, M., Yokoyama, N., Sako, Y., Fukunaga, M. and Ito, A. (2002b). The complete mitochondrial DNA sequence of the cestode Echinococcus multilocularis (Cyclophyllidea: Taeniidae). Mitochondrion 1, 497-509.

Nylander, J. A., Ronquist, F., Huelsenbeck, J. P. and Nieves-Aldrey, J. L. (2004). Bayesian phylogenetic analysis of combined data. Systematic Biology 53, 47-67.

Ortlepp, R.J. (1937). South African Helminths. Part I. The Onderstepoort Journal of Veterinary Science and Animal Industry 9, 311-336.

Posada, D. and Crandall, K. A. (1998). MODELTEST: testing the model of DNA substitution. Bioinformatics 14, 817-818.

Raush, R. L. (1967). A consideration of infraspecific categories in the genus Echinococcus Rudolphi, 1801 (Cestoda: Taeniidae). The Journal of 
Parasitology 53, 484-491.

Rausch, R. L. and Bernstein, J. J. (1972). Echinococcus vogeli sp. n. (Cestoda: Taeniidae) from the bush dog, Speothos venaticus (Lund). Zeitschrift für Tropenmedizin und Parasitologie 23, 25-34.

Rice, P., Longden, I. and Bleasby, A. (2000). EMBOSS: The European Molecular Biology Open Software Suite. Trends in Genetics 16, 276-277.

Ronquist, F. and Huelsenbeck, J. P. (2003). MrBayes 3: Bayesian phylogenetic inference under mixed models. Bioinformatics 19, 1572-1574.

Scott, J. C., Stefaniak, J., Pawlowski, Z. S. and McManus, D. P. (1997). Molecular genetic analysis of human cystic hydatid cases from Poland: identification of a new genotypic group (G9) of Echinococcus granulosus. Parasitology 114, 37-43.

Smyth, J. D. and Smyth, M. M. (1964). Natural and experimental hosts of Echinococcus granulosus and E. multilocularis, with comments on the genetics of speciation in the genus Echinococcus. Parasitology 54, 493-514.

Sweatman, G. K. and Williams, R. J. (1963). Comparative studies on the biology and morphology of Echinococcus granulosus from domestic livestock, moose and reindeer. Parasitology 53, 339-390.

Swofford, D. L. (2002). PAUP*: phylogenetic analysis using parsimony (and other methods) 4.0 beta. Sinauer Associates, Sunderland, Massachusetts, USA.

Telford, M. J., Herniou, E. A, Russell, R. B. and Littlewood, D. T. (2000). Changes in mitochondrial genetic codes as phylogenetic characters: two 
examples from the flatworms. Proceedings of the National Academy of Sciences USA 97, 11359-11364.

Thompson, J. D., Gibson, T. J., Plewniak, F., Jeanmougin, F. and Higgins, D. G. (1997). The Clustal_X windows interface: flexible strategies for multiple sequence alignment aided by quality analysis tools. Nucleic Acids Research 25, 4876-4882.

Thompson, R. C. A., Boxell, A. C., Ralston, B. J., Constantine, C. C., Hobbs, R. P., Shury, T. and Olson, M. E. (2006). Molecular and morphological characterization of Echinococcus in cervids from North America. Parasitology 132, 439-447.

Thompson, R. C. A. and McManus, D. P. (2003). Towards a taxonomic revision of the genus Echinococcus. Trends in Parasitology 18, 452-457.

Thompson, R. C. A., Lymbery, A. J. and Constantine, C. C. (1995). Variation in Echinococcus: towards a taxonomic revision of the genus. Advances in Parasitology 35, 145-176.

Verster, A. J. M. (1965). Review of Echinococcus species in South Africa. The Onderstepoort Journal of Veterinary Research 32, 7-118.

Wang, X., Tedford, R. H., van Valkenburgh, B. and Wayne, R. K. (2004). Ancestry: evolutionary history, molecular systematics, and evolutionary ecology of Canidae. In Biology and Conservation of Wild Canids (ed. MacDonald, D. W. \& Sillero-Zubiri, C.), pp. 39-54. Oxford University Press, Oxford.

Wayne, R. K., Geffen, E., Girman, D. J., Koepfli, K. P., Lau, L. M. and Marshall, C. R. (1997). Molecular systematics of the Canidae. 
Systematic Biology 46, 622-653.

Webster, G. A. and Cameron, T. W. M. (1961). Observations on experimental infections with Echinococcus in rodents. Canadian Journal of Zoology 39, 877-891.

Williams, R. J. and Sweatman, G. K. (1963). On the transmission, biology and morphology of Echinococcus granulosus equinus, a new subspecies of hydatid tapeworm in horses in Great Britain. Parasitology 53, 391-407.

Xiao, N., Qiu, J., Nakao, M., Li, T., Yang, W., Chen, X., Schantz, P. M., Craig, P. S. and Ito, A. (2005). Echinococcus shiquicus n. sp., a taeniid cestode from Tibetan fox and plateau pika in China. International Journal for Parasitology 35, 693-701. 


\section{Figure legends}

Fig. 1. Similarity of nucleotide sequences among mitochondrial genomes of 10 Echinococcus taxa. The positions of genes and non-coding regions are marked with their abbreviations. Shaded quadrates indicate genes for transfer RNA. Abbreviations: atp6, ATPase subunit 6; $\operatorname{cox} 1$ to 3, cytochrome $c$ oxidase subunits 1 to 3 ; nad1 to 6 and nad4L, NADH dehydrogenase subunits 1 to 6 and 4L; NCR, non-coding region; $r r n L$ and $r r n S$, large and small subunit ribosomal RNAs.

Fig. 2. The average frequencies of amino acid replacements in all mitochondrial proteins among 10 Echinococcus taxa. The diameter of the largest circle corresponds to 278 changes and other circles are scaled in diameter proportionally. Open circles represent the frequent changes more than 70. The values were determined from the alignment of amino acid sequences by MacClade 4.08 using the cladogram obtained by ML analysis.

Fig. 3. Phylogenetic trees for members of the genus Echinococcus inferred from mitochondrial genome data. Abbreviations: Eequ, E. equinus; Egra, E. granulosus sensu stricto; Emul, E. multilocularis; Eoli, E. oligarthrus; Eort, E. ortleppi; Eshi, E. shiquicus; Evog, E. vogeli; G6 to G8, Echinococcus genotypes; Tsol, Taenia solium (outgroup). (A) Cladogram obtained through maximum likelihood (ML) and partitioned Bayesian (PB) analyses. ML bootstrap proportions (\%) are shown above branches, while Bayesian posterior 
probabilities (\%) are shown below. The confidence values of both analyses using the nucleotide data sets \#1 and \#2 are shown in boldface. Numbers in italics represent the values obtained from the amino acid data set \#3. (B) Phylogram obtained by ML analysis using the data set \#1. The scale bar represents the estimated number of nucleotide substitutions per nucleotide site. Bold lines indicate the topology of Echinococcus using Canidae as definitive hosts. The orders of intermediate hosts (Ar, Artiodactyla; La, Lagomorpha; Pe, Perissodactyla; Ro, Rodentia) are mapped to branches.

Fig. 4. The proposed movements of ancient definitive hosts of Echinococcus. The double arrows on the Bering Strait show the dispersals of primitive canids and felids during the late Miocene. The single arrow on Central America represents the immigration of carnivores into South America during the early Pleistocene. 